\title{
A Case Series Of Arthritis In Hansen's Disease
}

\author{
Hendra Gunawan ${ }^{1 *}$, Deasy Natalia Adriana ${ }^{1}$, Awalia ${ }^{1}$, Joewono Soeroso ${ }^{1}$ \\ ${ }^{1}$ Department of Internal Medicine, Faculty of Medicine, Universitas Airlangga, Surabaya, Indonesia - Dr. Soetomo General \\ Hospital Surabaya, Indonesia
}

\section{A R T I C L E I N F O}

\section{Article history:}

Received 23 February 2020

Received in revised form 25 May 2020

Accepted 04 June 2020

Available online 31 October 2020

\section{Keywords:}

Hansen's disease,

leprosy,

arthritis,

lepra reactions.

*) Corresponding author:

sylvester@gmail.com

\begin{abstract}
A B S T R A C T
Hansen's disease or leprosy is a chronic granulomatous infectious disease caused by Mycobacterium leprae. Musculoskeletal complaints might be the initial presentation of patients with Hansen's disease. Symptoms are common with clinical spectrum ranging from mild to debilitating arthritis. Regarding its wide clinical spectrum, a history taking especially in an endemic area like Indonesia on the possibility of Hansen's disease cannot be ruled out in patients with arthritis. We reported 3 cases of arthritis in Hansen's disease. First case was a 62-year-old Javanese female who had a deformity and chronic poly symmetry arthritis. Second case was a 45-year-old Javanese male with chronic bilateral knee pain as initial presentation of Hansen's disease and the last case was 61-yearold Javanese male who had acute bilateral knee pain with erythematous maculae associated with Erythema Nodosum Leprosum. All cases responded to corticosteroid $1 \mathrm{mg} / \mathrm{BW} /$ day.
\end{abstract}

\section{Introduction}

Hansen's disease or leprosy is a chronic granulomatous infectious disease caused by Mycobacterium leprae that becomes a worldwide health problem. World Health Organization (WHO) reported that leprosy new cases were found in 115 countries among different WHO regions. WHO reported that Indonesia had 18.994 new leprosy cases in 2012. ${ }^{1}$

Leprosy's manifestations depend on the number of bacilli and host immune response. Ridley and Jopling classified leprosy as tuberculoid (TT), borderline tuberculoid (BT), borderline (BB), borderline lepromatous (BL), and lepromatous leprosy (LL). The classification is based on the host immune system with TT type is associated with strong cellular immune response to $\mathrm{M}$. leprae, LL type is associated with complete absence of cellular immune response to $\mathrm{M}$. leprae, and borderline type is associated with unstable immune response between TT and LL type. WHO classified leprosy as paucibacillary (TT and BT type) and multibacillary (BB, BL, and LL type) for treatment purpose..$^{2-4}$

Leprosy has a wide clinical manifestations ranging from cutaneous, neurological, to musculoskeletal symptoms. Musculoskeletal symptoms are the third most common clinical manifestations and it may present as the chief complaint. However, the wide range manifestations make it underdiagnosed. All types of leprosy may cause arthritis, therefore patient with leprosy should be evaluated thoroughly to prevent the detrimental, loss of function, and deformities that may occur as a complication. ${ }^{5}$

We present three cases of leprosy with arthritis. The first case is a middle-aged woman with LL and arthritis due to Erythema Nodosum Leprosum (ENL) and deformities of the PIP joints. The second and third case showed two men with arthritis on knees as an initial presentation of multibacillary leprosy with ENL reaction.

\section{Case report}

Case 1

A 62 years old Javanese female came to clinic with pain on her left knee worsening in 2 weeks. She also complained that the rashes on face and extremities became tender and painful. She had fever and malaise for a week with inability to clench her fingers due to swelling for 9 months. Morning stiffness was felt for 30 minutes. She took multibacillary leprosy and taking MDT drugs comprises of rifampicin, clofazimine, and dapsone for 6 months. On physical examination, she was alert; with Visual Analog Scale (VAS) 5, stable hemodynamic and axillary temperature was $38,70 \mathrm{C}$. There were tender erythematous maculae on her face, neck, and extremities. Her gait seemed normal, but deformities (swan neck) and edema in both hands as well as 
fingers were found (left side was more prominent) (Figure 1) as well as joint effusion and tenderness in her left knee. Blood test showed elevated Erythrocyte Sedimentation Rate (ESR) $80 \mathrm{~mm} / \mathrm{h}$. Radiology examination of the hands showed the narrowing joint space in right Proximal Interphalangeal joints (PIP) II-III-IV with no joint erosion. Her initial assessment was leprosy (MB type), ENL, acute arthritis of the left knee, and swan neck deformities of the hands. Joint aspiration of the left knee was done but the M. leprae was not found. She was given intravenous methylprednisolone $1 \mathrm{mg} / \mathrm{kgBW}$, oral MDT, and acetaminophen $500 \mathrm{mg}$ for 3 days and tapered into $0,5 \mathrm{mg} /$ $\mathrm{kgBW} /$ day oral methylprednisolone for 3 days by the time she was discharged.

\section{Case 2}

A 45 year old Javanese male came into emergency department due to fever and bloody vomiting since 3 days earlier. Fever occurred mostly at night but then became continuous. He had a history of taking piroxicam and herbal medication for his left knee pain. Pain was felt on both knees for 9 months and steadily worsened with palpated tenderness since a week ago. He also revealed that his face became reddish and his eyebrows gradually fell out since 6 months ago. He denied any history of leprosy nor receiving medication for his complaints. He was alert with stable hemodynamic. Axillary temperature appeared 39,30C and VAS 4. He appeared anemic with madarosis and diffuse erythematous maculae on his face. Erythematous maculae along with hypopigmented hypoesthesia maculae were also found on both forearms along with bilateral thickening of ulnar nerves.

Initial blood test showed hemoglobin $8.5 \mathrm{~g} / \mathrm{dL}$, leukocytes 10,600 cells/mm3, neutrophil $73.2 \%$, ESR $120 \mathrm{~mm} / \mathrm{h}$. His hemoglobin increased to $11.5 \mathrm{~g} / \mathrm{dL}$ after transfusion with packed red cells (PRC), C-reactive Protein (CRP) 4,5 mg/dL, Rheumatoid Factor (RF) negative, and anti-cyclic citrullinated peptides (anti-CCP) negative.

His chest and knee radiology examination were normal. Endoscopy examination revealed an erosive gastritis. Arthrocentesis of the knee showed no presentation of acid fast bacteria. Slit smear of the ear however revealed acid fast bacteria with Bacterial Index (BI) +4 and Morphological index (MI) 70\%. His initial assessment was haematemesismelaena due to erosive gastritis, leprosy (MB type), ENL, and bilateral knee arthritis. Two bags of PRC transfusion with $80 \mathrm{mg}$ Omeprazole injections were given as initial treatments. Intravenous methylprednisolone was given with dose $1 \mathrm{mg} / \mathrm{kgBW}$ and $500 \mathrm{mg}$ acetaminophen thrice a day. His symptoms improved in day-5 and was discharge with VAS 2 and ESR decreased to $70 \mathrm{~mm} / \mathrm{h}$, methylprednisolone then was changed into oral $0,5 \mathrm{~kg} / \mathrm{BW}$ along with MDT.

\section{Case 3}

A 61-year-old, Javanese male came into emergency department due to bilateral knees pain for 2 weeks. Pain on both knees was sharp, steadily worse, and did not improve with rest. Fever was present for 3 days. He also had reddish spot on his face, bodies, and left knee since 2 months ago along with the loss of eyebrows. He denied any history of leprosy and medication for his pain. He was alert with stable hemodynamic. Axillary temperature was 39,70C and VAS 6. Warm and tender erythematous maculae were found on head, chest, abdomen, knee, and feet. (Figure 2). Madarosis and tender bilateral knee joint effusions were found on palpation.

Initial blood examination showed hemoglobin $10.2 \mathrm{~g} /$ dL, leukocytes 22,700 cells/mm3, neutrophil 93.6\%, ESR $90 \mathrm{~mm} / \mathrm{h}, \mathrm{CRP} 2.5 \mathrm{mg} / \mathrm{dL}$, RF negative, anti-CCP negative.

His chest and knee X-rays were normal. Slit smears of the ear revealed the presence of acid-fast bacteria with BI +2 and MI 75\%. Arthrocentesis of knee revealed transudate without acid fast bacteria nor other bacterias. His initial assessment was sepsis due to septic arthritis. He was diagnosed as leprosy (MB type) with ENL according to the results of slit smears revealed acid fast bacteria. Therefore, intravenous methylprednisolone $1 \mathrm{mg} / \mathrm{kgBW}$ was given. His symptoms improved within day 6 of hospitalization. Thus patient discharged, methylprednisolone was changed to oral at $0,5 \mathrm{mg} / \mathrm{kgBW}$ and MDT was initiated.

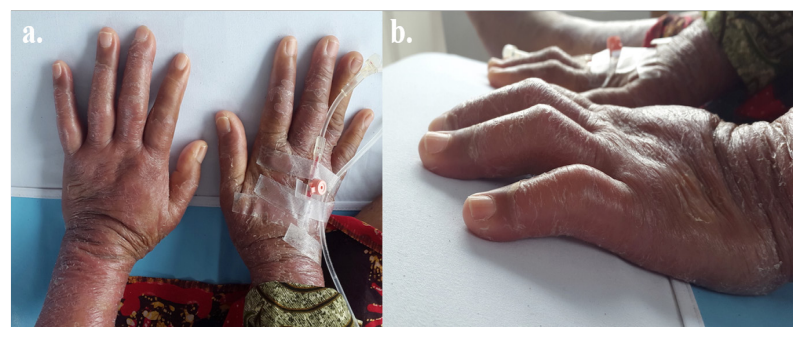

Figure 1. Case 1. a. Edema in both hands. Left side prominent (arrow). B. Swan neck deformities in both hands (arrows).

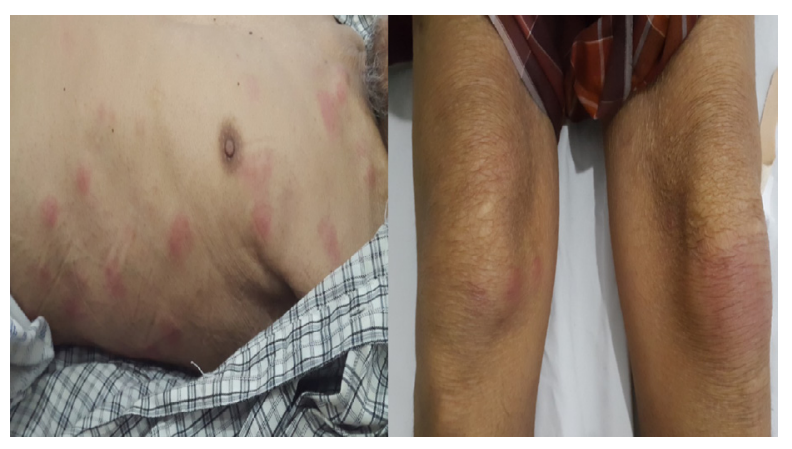

Figure 2. Case 3. a. Warm and tender erythematous maculae (arrows). B. Bilateral knee joint effusions (arrows).

\section{Discussion}

Most Leprosy's musculoskeletal manifestations may resemble other disorders making them often underdiagnosed or misdiagnosed as other diseases such as rheumatic arthritis (RA) and systemic lupus erythematosus (SLE).$^{6-8}$ The presence of fever, fatigue, paresthesia, skin lesions, musculoskeletal complaints may resemble connective tissue diseases such as SLE, RA, dermatopolymyositis, and systemic vasculitis. Systemic manifestations are more commonly found in MB. The presence of RF, anti-CCP, anti-neutrophil cytoplasmic antibodies, and anticardiolipin antibodies may be observed. ${ }^{9}$

There are type I and type II of leprosy reactions based on T-cell reactivity. Acute inflammation is found in type I reaction. Type I reaction is also a delayed 
hypersensitivity reaction and can occur many years after completion of MDT.5,10Type II leprosy reaction or ENL is an immune complex-mediated reaction. It is mainly found in borderline lepromatous or lepromatous leprosy. Neutrophil infiltration and activation of complement cascades are often found in type II leprosy reaction. Elevated TNF- $\alpha$ and $\mathrm{TH} 2$ cytokine profile are prominent in ENL. 5,10

Arthritis may be the initial symptom in leprosy and mainly occurs in MB type. The pathogenesis of arthritis in leprosy is not fully understood due to the similarities of its clinical manifestations. The proposed mechanisms are reactional state (granulomatous or ENL reaction) or direct infiltration and proliferation of M. leprae in the synovium leading to peripheral joint neuropathy and finally joint destruction. ${ }^{5,6}$ There is no formal classification of arthritis in leprosy, but it can be divided into (1) Charcot's joints, (2) septic arthritis (post-traumatic nonspecific), (3) specific arthritis due to the infiltration of M. leprae, (4) acute polyarthritis of lepra reaction, and (5) chronic arthritis..$^{5,9}$

Acute arthritis is a common manifestation in type I and II leprosy reaction. Acute arthritis in leprosy may resemble RA. Its manifestations range from symmetrical polyarthritis affecting small joints to the knees, ankles, shoulders, and elbows though the later are less common. Shejpal and Henriques et al. have reported the arthritis of leprosy reactions resembling RA. The differences between arthritis in leprosy and RA are (1) the absence of rheumatoid factor and antiCCP, (2) arthritis in leprosy remains non-erosive, (3) no improvement with the introduction of RA therapy such as DMARDs and corticosteroids, and (4) the appearance of skin lesions may present in leprosy patients. Most patients with acute arthritis in leprosy will resolve in 4 weeks. ${ }^{5,7}$ Recent studies demonstrated the presence of anti-CCP antibodies, known for its high specificity to $\mathrm{RA}$, in various infectious diseases such as tuberculosis, leishmaniasis, and leprosy with one study reported that $11 \%$ of leprosy patients were positive for anti-CCP. It should be noted that RF may be positive in $4,5-15 \%$ of subjects with leprosy, especially LL type. ${ }^{11}$ However, in all cases, joint erosions were not found in radiology and laboratory examinations showed the absence of M.leprae, RF, and anti-CCP.

Chronic arthritis may be found in leprosy and may be associated with both of leprosy reactions. Atkin et al. were the first to documented chronic arthritis without reactional states. Chronic arthritis not associated with reactional states' had gradual onset, encompassed whole range of leprosy, and had no other features of leprosy reactional states. Permanent joint deformities especially in hands such as boutonniere, swan neck, mallet finger, and ulnar drift may occur in leprosy patients which make the diagnosis of leprosy and RA more difficult. The risk of chronic arthritis increased in line with the durations of leprosy. ${ }^{5}$ It associated with reactional states may represent as "Arthus" reaction or a reactive arthropathy and it was restricted to the lepromatous type of leprosy. ${ }^{12}$

We presented a case series of arthritis leprae due to chronic leprosy (case 1) and lepra reactions (all cases).
In case 1 , the swan neck deformities which caused the disability were due to chronic leprosy. We considered acute arthritis due to lepra reactions in all cases due to the gradually increasing pain on knees (unilateral in case 1 and bilateral in case 2 and 3) accompanied with ENL. It is in line with the previous publications which reported that most patients with ENL complained of joint pain; however objective evidence of arthritis was definitely present in only one third of the cases.13 Pereira et al., reported that $1,9 \%$ patients presented only arthralgia, and 4,4\% presented with arthritis. They also reported that arthritis in leprosy can occur in form of monoarticular (7,3\%), oligoarticular $(36,3 \%)$, and polyarticular (56,3\%).14 Therefore, in both case 2 and 3 , it is an monoarticular arthritis which are less frequent form of arthritis in leprosy.

The management of arthritis in leprosy depends on the presence of lepra reactions. Therefore, treatment strategy should be focused to control acute inflammation, ease pain, and reverse nerve damage. ${ }^{15}$ Corticosteroid should be added with MDT in leprosy reaction. The starting dose of corticosteroid is $1 \mathrm{mg}$ / $\mathrm{kgBW} /$ day equivalent prednisone The duration of corticosteroid therapy with tapered dose ranges from 4-6 months. In our cases, all patients were in ENL state, therefore methylprednisolone i.v $1 \mathrm{mg} / \mathrm{kgBW} /$ day was given. All patient's general condition improved in 3-6 days. Pain, joint effusion, and swelling were alleviated and methylprednisolone was changed into oral $0,5 \mathrm{mg}$ / $\mathrm{kgBW} /$ day. Tapering down was done by reducing the total daily methylprednisolone dosage by $1 / 4$ once in 4 days. 16

If corticosteroid and MDT could not control inflammation, clofazimine, thalidomide or biologic agents (TNF- $\alpha$ inhibitor) might be considered.5,9 The consistent over-expression of TNF- $\alpha$ in patients with severe ENL provided the rationale for the use of TNFinhibitory agents. ${ }^{17,18}$

\section{Conclusion}

A case series of patients with arthritis in leprosy had been reported. The diagnosis of leprosy may pose a challenge especially in non-endemic area. Type I and II leprosy reactions might have acute arthritis as its manifestations. Acute arthritis in leprosy may resemble RA and prompt history taking as well as examinations should be performed meticulously. Corticosteroid and MDT are the backbone of arthritis associated with ENL and should be given to control inflammation. Anti TNF- $\square$ should be considered in difficult reactions.

\section{Conflict of Interest}

The author stated there is no conflict of interest

\section{References}

1.World Health Organization. Global leprosy: update on the 2012 situation. Weekly Epidemiological Report. 2013;35(88):365-80.

2.Degang Y, Nakamura K, Akama T, et al. Leprosy as a Model of Immunity. Future Microbiology. 2014;9(1):43-54.

3. Walker S, Lockwood D. The clinical and immunological features of leprosy. British Medical Bulletin. 2006;77(78):103-21.

4.Pardillo EF, Fajardo TT, Abalos RM, Scollard D, Gelber RH. Methods for the Classifcation of Leprosy for treatment purposes. Clinical 
Infectious Diseases. 2007;44(15):1096-9.

5.Chauhan S, Wakhlu A, Agarwal V. Arthritis in Leprosy. Rheumatology. 2010;49(1):2237-42.

6.de Oliveira Fernandes TRM, Espindola MMM, Korinfskin JP, de Oliveira Correa LM. Arthritis and diagnosis of leprosy: a case report and review of the literature. Anais Brasileiros de Dermatologia. 2014;89(2):323-5.

7.Henriques CC, Lopez B, Mestre T, Grima B, Panarra A, Riso N. Leprosy and Rheumatoid Arthritis: Consequence or Association? Blackwell Medical Journal Case Reports. 2012;47(1):1-4.

8.Shejpal A. Hansen's Arthritis: An Overlooked Entity. Vidarbha Journal of Internal Medicine. 2014;16:43-5.

9.Ribeiro SLE, Guedes EL, Pereira HLA, de Souza LS. Systemic Manifestations and Ulcerative Skin Lesion in Leprosy: Differential Diagnosis with Rheumatic Diseases. Brasilian Journal of Rheumatology. 2009;49(5):623-9.

10.Kahawita IP, Walker S, Lockwood D. Leprosy type 1 reactions and erythema nodosum leprosum. Anais Brasilieros de Dermatologia. 2008;83(1):75-82

11.Zavala-Cerna MG, Fafutis-Morris m, Guillen-Vargas C, et al. Anticyclic citrullinated peptide antibodies and rheumatoid factor sera titers in leprosy patients from Mexico. Rheumatology International. 2012;2012(32):3531-6.
12.Atkin S, El-Ghobarey A, Kamel M, Owen J, DIck W. Clinical and Laboratory Studies of Arthritis in Leprosy. Blackwell Medical Journal. 1989;298(1):1423-5.

13.Albert D, Weisman M, Kaplan R. The Rheumatic Manifestations of leprosy (Hansen disease). Medicine. 1980;59(6):442-8.

14.Pereira HLA, Ribeiro SLE, Pennini S, Sato EI. Leprosy-related joint involvement. Clinical Rheumatology. 2009;2009(28):79-84.

15.Helling CA, Locursio A, Manzur ME, Leticia M, de Fonseca S. Remitting seronegative symmetrical synovitis with pitting edema in leprosy. Clinical Rheumatology. 2006;25(12):95-7.

16.Longui CA. Glucocorticoid therapy: minimizing side effect. Jornal de Pediatria. 2007;83(Supp.5):S163-71.

17.Ramien ML, Wong A, Keystone JS. Severe Refractory Erythema Nodosum Leprosum Successfully Treated with the Tumor Necrosis Factor Inhibitor Etanercept. Clinical Infectious Diseases. 2011;52(5):e133-5.

18.Faber WR, Jensema AJ, Goldschmidt WF. Treatment of Recurrent Erythema Nodosum Leprosum with Infliximab. The New England Journal of Medicine. 2006;355(7):739. 